\title{
Radial solutions for fully nonlinear elliptic equations of Monge-Ampère type
}

\author{
Limei Dai ${ }^{1}$, Huihui Cheng ${ }^{2 *}$ and Hongfei $\mathrm{Li}^{3}$
}

\section{"Correspondence:}

chenghuihui@ncwu.edu.cn

${ }^{2}$ School of Mathematics and

Statistics, North China University of

Water Resources and Electric Power,

Zhengzhou, China

Full list of author information is

available at the end of the article

\begin{abstract}
First, the symmetry of classical solutions to the Monge-Ampère-type equations is obtained by the moving plane method. Then, the existence and nonexistence of radial solutions in a ball are got from the symmetry results. Finally, the existence and nonexistence of classical solutions to Hessian equations in bounded domains are considered.
\end{abstract}

Keywords: Monge-Ampère-type equations; Hessian equations; Radial symmetry

\section{Introduction}

We firstly consider the Monge-Ampère-type equations

$$
\mathcal{F}(u)=\operatorname{det}\left[D^{2} u+M(x, u, D u)\right]=g(x, u, D u),
$$

with $D^{2} u$ being the Hessian matrice of $u, M(x, u, D u)$ being a given symmetric matrix function, and $g$ being a positive function. In (1.1), the operator $\mathcal{F}$ is elliptic for $u$ if $D^{2} u+M(x, u, D u)>0$, and at the same time, a $C^{2}$-solution $u$ is called an elliptic solution.

Equations of the form (1.1) come from geometric optics [1], optimal transportation [2], conformal geometry [3], isometric embedding [4], and reflector-refractor problems [58]. In optimal transportation, the function $u$ is called the potential function and it satisfies the optimal transportation equation

$$
\operatorname{det}\left[D^{2} u-D_{x}^{2} \tilde{\theta}(x, Y(x, D u))\right]=\tilde{\eta} /\left|\operatorname{det} Y_{p}\right|
$$

with the vector field $Y: D \times \mathbb{R} \times \mathbb{R}^{n} \rightarrow \mathbb{R}^{n}, Y=Y(x, z, p)$ being independent of $z$, $D$ being a domain in $\mathbb{R}^{n}$, det $Y_{p} \neq 0, Y$ coming from a cost function $\tilde{\theta}: \mathbb{R}^{n} \times \mathbb{R}^{n} \rightarrow \mathbb{R}, \tilde{\theta}=\tilde{\theta}(x, y)$ being determined by the equations

$$
\tilde{\theta}_{x}(x, Y(x, p))=p,
$$

and $\tilde{\eta}$ being a known nonnegative function $\tilde{\eta}: D \times \mathbb{R} \times \mathbb{R}^{n} \rightarrow \mathbb{R}$. The optimal transportation equation is of the form (1.1).

(c) The Author(s) 2021. This article is licensed under a Creative Commons Attribution 4.0 International License, which permits use, sharing, adaptation, distribution and reproduction in any medium or format, as long as you give appropriate credit to the original author(s) and the source, provide a link to the Creative Commons licence, and indicate if changes were made. The images or other third party material in this article are included in the article's Creative Commons licence, unless indicated otherwise in a credit line to the material. If material is not included in the article's Creative Commons licence and your intended use is not permitted by statutory regulation or exceeds the permitted use, you will need to obtain permission directly from the copyright holder. To view a copy of this licence, visit http://creativecommons.org/licenses/by/4.0/. 
Monge-Ampère-type equations have got a lot of interest $[9,10]$. For $M \equiv 0$, the equation (1.1) becomes the standard Monge-Ampère equation $\operatorname{det} D^{2} u=g(x, u, D u)$ which was investigated in [11-13], etc. For instance, the a priori estimates and existence of solutions [11], as well as the symmetry, existence, and nonexistence of solutions [13] were studied. The blow-up solutions to the Monge-Ampère equation and fully nonlinear equations and convex solutions of the Monge-Ampère systems can be found in [14-16]. If $M \neq 0$ and $M$ is linear in, or independent of, the gradient $D u$ [4], one has very similar a priori estimates as those of the standard Monge-Ampère equations. But if $M$ is nonlinear in $D u$, the situation is very different. The interior a priori estimates of (1.1) were got in [8] for dimension two, and in [1] for all dimensions. The interior $C^{2}$-estimates and the interior regularity of (1.1) were obtained by Ma-Trudinger-Wang [17] under some structure conditions on the matrix $M$ and under a generalized target convexity condition. Under weaker conditions, Trudinger-Wang [18] have proved the global $C^{2}$-estimates and regularity to the second boundary value problem of (1.1). However, the $C^{2, \alpha}$-estimates are few. When $M=M(x, D u), g=g(x)$, Liu-Trudinger-Wang [19] have proved the interior $C^{2, \alpha}$-estimates of (1.1) under appropriate assumptions and Huang-Jiang-Liu [20] have got the boundary $C^{2, \alpha}$-estimates of solutions. Jiang-Trudinger-Yang [21] have proved that the Dirichlet problem of (1.1) has a unique classical solution. Dai and Li [22] obtained the necessary and sufficient condition for the existence of subsolutions to (1.1) with $M=\kappa I, \kappa \geq 0$, and $g=g(u)$.

When studying partial differential equations, we are interested in knowing whether the solutions are symmetric about some plane, or monotone in some direction. Gidas-NiNirenberg [23] obtained the first symmetry results. Utilizing the moving plane method, they showed that if the solutions to the Dirichlet problem of $-\Delta u=g(u), g \in C^{0,1}(\mathbb{R})$ in a ball are $C^{2}$ and positive, then they are symmetric. Alexandrov [24] first introduced the distinguished the moving plane method and then Serrin [25] developed it. Later, many authors generalized the symmetry results. Especially, Li [26] extended the symmetry results to fully nonlinear elliptic equations $G\left(x, u, D u, D^{2} u\right)=0$, with $G$ being uniformly elliptic on smooth domains, and the author of [27] studied the symmetry of viscosity solutions to fully nonlinear parabolic equations $-u_{t}+G\left(x, t, u, D u, D^{2} u\right)=0$ in a singular domain. Zhang-Wang [13] have obtained that the classical solutions to the standard MongeAmpère equations $\operatorname{det} D^{2} u=e^{-u}$ are symmetric.

Let $D \subset \mathbb{R}^{n}$ be a domain. Consider the symmetry of solutions for the Dirichlet problem

$$
\begin{cases}\operatorname{det}\left[D^{2} u+M(x)\right]=g(u), & x \in D, \\ u=0, & x \in \partial D .\end{cases}
$$

We suppose that $0 \in D$ and the following hypotheses hold:

(F) $g \geq g_{0}>0, g_{0}$ is a constant, $g$ is Lipschitz continuous in $u$.

(M) $M(x)=\left(m_{i j}(x)\right), m_{i j}(x), i, j \in\{1,2, \ldots, n\}$ are sufficiently smooth functions, $M(x)$ and $D^{2} u+M(x)$ are positive definite, and $\operatorname{det}[M(x)]<g_{0}$ for $x \in D$.

Let

$$
x^{t}=\left(2 t-x_{1}, x_{2}, \ldots, x_{n}\right) .
$$


We assume that $m_{i j}(x), i, j \in\{1,2, \ldots, n\}$ satisfy the following hypotheses:

$$
\begin{aligned}
& \left(H_{1}\right) \quad m_{i j}\left(x^{t}\right)=m_{i j}(x), \quad i, j \in\{2, \ldots, n\}, \\
& m_{11}\left(x^{t}\right)=m_{11}(x) \\
& m_{1 j}\left(x^{t}\right)=-m_{1 j}(x), \quad m_{j 1}\left(x^{t}\right)=-m_{j 1}(x), \quad j=2, \ldots, n, \quad \text { i.e. }, \\
& \left(m_{i j}\left(x^{t}\right)\right)=\left(\begin{array}{cccc}
m_{11}(x) & -m_{12}(x) & \ldots & -m_{1 n}(x) \\
-m_{21}(x) & m_{22}(x) & \ldots & m_{2 n}(x) \\
\vdots & \vdots & \ddots & \vdots \\
-m_{n 1}(x) & m_{n 2}(x) & \ldots & m_{n n}(x)
\end{array}\right)
\end{aligned}
$$

The symmetry results are the following.

Theorem 1.1 Let the hypotheses $(\mathbf{F})$ and $(\mathbf{M})$ hold. Suppose that $D \subset \mathbb{R}^{n}$ is a bounded domain, convex in the $x_{1}$ direction, symmetric about the hyperplane $\left\{x: x_{1}=0\right\}$, and its boundary $\partial D$ is smooth. Then any solution $u \in C^{3}(\bar{D})$ of the Dirichlet problem (1.2) is also symmetric about the hyperplane $\left\{x: x_{1}=0\right\}$. In addition, for $x_{1}<0, u$ is decreasing in $x_{1}$.

Now we discuss the existence and nonexistence of classical solutions for the equations with exponential terms on the right-hand side which usually appear in geometry. Consider

$$
\begin{cases}\operatorname{det}\left(D^{2} u+\kappa I\right)=e^{-u}, & x \in B_{H}(0), \\ u=0, & x \in \partial B_{H},\end{cases}
$$

where $\kappa>0$ is a constant, $I$ is the identity matrix, and $B_{H}=B_{H}(0)$ is the ball centered at the origin and having radius $H$.

\section{Theorem 1.2}

(1) The Dirichlet problem (1.3) has no solution if $0<\kappa<1$ and $H$ is large enough.

(2) The Dirichlet problem (1.3) has a unique elliptic solution if $0<\kappa<e$ and $H$ is small.

(3) The Dirichlet problem (1.3) has a unique elliptic solution if $\kappa \geq e$ and $0<H<+\infty$ is arbitrary.

Let $D \subset \mathbb{R}^{n}$ be a bounded domain and $0 \in D$. In the following, we will consider the Hessian equation

$$
S_{k}\left(D^{2} u\right)=\sigma_{k}\left(\lambda\left(D^{2} u\right)\right)=g \quad \text { in } D
$$

where $\lambda\left(D^{2} u\right)=\left(\lambda_{1}, \ldots, \lambda_{n}\right)$ is the eigenvalues of $D^{2} u$,

$$
\sigma_{k}\left(\lambda\left(D^{2} u\right)\right)=\sum_{j_{1}<\cdots<j_{k}} \lambda_{j_{1}} \cdots \lambda_{j_{k}}
$$

is the $k$ th elementary symmetric function of $\lambda\left(D^{2} u\right), k=1, \ldots, n$ and $g$ is a known positive function of $D$.

Notice that if $k=1$, equation (1.4) corresponds to $\Delta u=g(u)$, which is semilinear, and if $k=n$, then we get $\operatorname{det} D^{2} u=g(u)$, which is the standard Monge-Ampère equation which 
is fully nonlinear. For $1<k<n$, equation (1.4) is fully nonlinear and elliptic, and it is important in conformal geometry, etc., see [28]. We will restrict the class of functions to guarantee that (1.4) is elliptic. Let $u \in C^{2}(D)$ and $\Gamma_{k}=\left\{\lambda \in \mathbb{R}^{n} \mid \sigma_{l}(\lambda)>0, l=1, \ldots, k\right\}$ be a convex cone, and let its vertex be the origin. If $\lambda\left(D^{2} u\right) \in \Gamma_{k}$, then $u$ is said to be uniformly $k$-convex. If $\lambda\left(D^{2} u\right) \in \overline{\Gamma_{k}}$, then $u$ is said to be $k$-convex. According to [28], $S_{k}\left(D^{2} u\right)$ is elliptic if $u$ is uniformly $k$-convex, that is,

$$
\left(S_{k}^{i j}\left(D^{2} u\right)\right) \equiv\left(\frac{\partial}{\partial r_{i j}} S_{k}\left(D^{2} u\right)\right)>0
$$

For any $y \in \partial D$, let $v(y)=\left(v_{1}, \ldots, v_{n-1}\right)$, where $v_{j}, j=1, \ldots, n-1$, represent the principal curvatures of $y \in \partial D$. If $v(y) \in \Gamma_{k-1}$, then the domain $D$ is said to be uniformly $(k-1)$ convex. We always assume that the domain $D$ in (1.4) is uniformly $(k-1)$-convex.

Equation (1.4) has seen a lot of investigation. If $g=g(x)$, independent of $u$, the existence of solutions is considered, see [28-30], among other sources. If $g=g(x, u)$, the multiplicity of (1.4) for $k=1$ and $k=n$ is investigated in [13, 31, 32]. For instance, Zhang-Wang [13] obtained the multiplicity of solutions for the Dirichlet problem of standard MongeAmpère equation $\operatorname{det} D^{2} u=e^{-u}$ and $\operatorname{det} D^{2} u=e^{\tau u}$. For $1<k<n$, Jacobsen [33] considered the multiplicity of solutions of

$$
\begin{cases}S_{k}\left(D^{2} u\right)=\lambda e^{-u}, & x \in D, \\ u=0, & x \in \partial D .\end{cases}
$$

The author of [34] studied the multiplicity of solutions of

$$
\begin{cases}S_{k}\left(D^{2} u\right)=\lambda g(x, u), & x \in D, \\ u=0, & x \in \partial D .\end{cases}
$$

Li [35] investigated the existence of classical solutions for the Dirichlet problem of the equations $\sigma_{k}^{\frac{1}{k}}\left(\lambda\left(u_{i j}(x)+\tilde{b}_{i j}(x)\right)\right)=\psi(x)$. Wang-Bao [36, 37] investigated the symmetry of solutions to $\sigma_{k}\left(\lambda\left(D^{2} u\right)\right)=g(x, u, D u)$.

In this paper, using the radial symmetry results for Hessian equations, we consider the following problem:

$$
\begin{cases}S_{k}\left(D^{2} u\right)=e^{-u}, & x \in D, \\ u=0, & x \in \partial D .\end{cases}
$$

Theorem 1.3 For $D=B_{H}(0)$, if $H>0$ is large enough, then (1.5) has no solution, and if $H>0$ is small enough, then (1.5) has a solution.

Letting $\bar{\alpha}$ be a parameter, we also consider the problem

$$
\begin{cases}S_{k}\left(D^{2} u\right)=e^{-\bar{\alpha} u}, & x \in D, \\ u=0, & x \in \partial D .\end{cases}
$$


Theorem 1.4 There exists some constant $\bar{\alpha}^{*}>0$ such that for any $\bar{\alpha}>\bar{\alpha}^{*}$, there does not exist any solution of (1.6).

We arrange the paper as follows: Some maximum principles of linear elliptic equations are proved in Sect. 2. We get the symmetry of solutions for problem (1.2) in Sect. 3. Theorems 1.1 and 1.2 will be demonstrated in Sect. 4, and Theorems 1.3 and 1.4 will be demonstrated in Sect. 5.

\section{Maximum principles for "narrow regions"}

Let $D \subset \mathbb{R}^{n}$ be a domain. In this section, a few maximum principles for the linear elliptic equation

$$
-a^{i j}(x) D_{i j} u+\theta(x) u=0 \quad \text { in } D
$$

will be given. These maximum principles may be found in other references, but here for convenience we list them and give their proofs. We always assume in this section that $a^{i j}(x)$, $\theta(x)$ are continuous and bounded in $\bar{D}$, and $\left(a^{i j}(x)\right)$ is uniformly elliptic, that is, there are some constants $0<\lambda \leq \Lambda$ such that

$$
\Lambda|\xi|^{2} \geq a^{i j}(x) \xi_{i} \xi_{j} \geq \lambda|\xi|^{2}, \quad \xi \in \mathbb{R}^{n}
$$

Lemma 2.1 Suppose that $D \subset \mathbb{R}^{n}$ is bounded. Let $\rho(x) \in C(\bar{D})$ and $h(x)$ be positive on $\bar{D}$ satisfying

$$
-a^{i j}(x) D_{i j} h+\rho(x) h \geq 0 .
$$

Suppose that $u \in C^{3}(\bar{D})$ satisfies

$$
\begin{aligned}
& -a^{i j}(x) D_{i j} u+\theta(x) u \geq 0, \quad x \in D, \\
& u \geq 0, \quad x \in \partial D .
\end{aligned}
$$

If

$$
\theta(x)>\rho(x), \quad \forall x \in D,
$$

then we have $u \geq 0$ in $D$.

Proof We use contradiction arguments to prove the lemma. Let $\bar{x} \in D$ be some point satisfying $u(\bar{x})<0$. Define

$$
q(x)=\frac{u(x)}{h(x)}, \quad x \in D .
$$

Then from the fact that $h(x)>0$ in $D$, we know that $q(\bar{x})<0$. Let $x_{0} \in D$ be one minimum point of $q(x)$, then $q\left(x_{0}\right)<0$. By a direct computation we get that

$$
D_{i} q=\frac{1}{h} D_{i} u-\frac{u}{h^{2}} D_{i} h,
$$




$$
D_{i j} q=\frac{1}{h} D_{i j} u-\frac{u}{h^{2}} D_{i j} h-\frac{1}{h} D_{i} q D_{j} h-\frac{1}{h} D_{j} q D_{i} h .
$$

Therefore, we obtain

$$
-a^{i j}(x) D_{i j} q=\frac{1}{h}\left(-a^{i j} D_{i j} u+\frac{a^{i j} D_{i j} h}{h} u\right)+a^{i j} \frac{1}{h} D_{i} q D_{j} h+a^{i j} \frac{1}{h} D_{j} q D_{i} h .
$$

On the one hand, since $q\left(x_{0}\right)$ is a minimum of $q(x)$, we have

$$
D q\left(x_{0}\right)=0 \quad \text { and } \quad D^{2} q\left(x_{0}\right) \geq 0 .
$$

Then

$$
-a^{i j}\left(x_{0}\right) D_{i j} q\left(x_{0}\right) \leq 0 .
$$

On the other hand, by (2.1)-(2.3), and considering $u\left(x_{0}\right)<0$, we get, at point $x_{0}$,

$$
\begin{aligned}
-a^{i j} D_{i j} u+\frac{a^{i j} D_{i j} h}{h} u & \geq-a^{i j} D_{i j} u+\rho(x) u \\
& >-a^{i j} D_{i j} u+\theta(x) u \\
& \geq 0 .
\end{aligned}
$$

By virtue of (2.4) and (2.5), we then have

$$
-a^{i j}\left(x_{0}\right) D_{i j} q\left(x_{0}\right)>0 \text {. }
$$

This contradicts (2.6), and the proof of Lemma 2.1 is completed.

Using the same idea of the above arguments, we can extend Lemma 2.1 to unbounded domains provided that $u$ is "nonnegative" at infinity.

Lemma 2.2 Let D be unbounded. If, in addition to the conditions in Lemma 2.1, we suppose further that

$$
\liminf _{|x| \rightarrow+\infty} u(x) \geq 0
$$

then $u(x) \geq 0, x \in D$.

Proof We consider the same $q(x)$ as above in the proof of Lemma 2.1. Obviously, assumption (2.7) means that we cannot obtain the minimum of $q(x)$ at infinity. So the minimum point of $q$ is in the interior of $D$. Similar to the proof of Lemma 2.1, we finish the proof of Lemma 2.2.

In the following, we give some particular $h(x)$ such that $\theta(x)$ satisfies (2.3) in the narrow regions, thus the maximum principle can be applied to those narrow regions. Let

$$
D=\left\{x \mid 0<x_{1}<l-2 \tilde{\varepsilon}, 0<\tilde{\varepsilon}<l / 2\right\}
$$


be a narrow region with width $l-2 \tilde{\varepsilon}$, then we can choose

$$
h(x)=\sin \left(\frac{x_{1}+\tilde{\varepsilon}}{l-\tilde{\varepsilon}}\right) .
$$

Then

$$
-a^{i j} D_{i j} h=\left(\frac{1}{l-\tilde{\varepsilon}}\right)^{2} a^{11} h .
$$

For the constants $\Lambda$ and $\lambda$, if $\left(a^{i j}(x)\right)$ is uniformly elliptic, let $\rho(x)=-\lambda / l^{2}$, so $\rho(x)$ can be very negative when $l$ is sufficiently small and

$$
-a^{i j} D_{i j} h=\left(\frac{1}{l-\tilde{\varepsilon}}\right)^{2} a^{11} h \geq \frac{\lambda}{l^{2}} h .
$$

So

$$
-a^{i j} D_{i j} h+\rho(x) h \geq 0
$$

Now applying Lemma 2.1, we have

Corollary 2.3 (Narrow region principle) Assume that $D$ is bounded and the width $l$ of $D$ is small enough. Suppose further that for the constants $\Lambda$ and $\lambda,\left(a^{i j}(x)\right)$ is uniformly elliptic and $u$ satisfies (2.2). Then $u \geq 0$ in $D$, provided that $u \geq 0$ on $\partial D$.

\section{Symmetry of solutions}

Lemma 3.1 The solution of (1.2) is negative in $D$, that is, $u<0$ in $D$.

Proof We firstly demonstrate that $u \leq 0$ in $D$. We use the contradiction arguments. Suppose this is not true, then there are some point $x_{0} \in D$ and its neighborhood $\mathcal{N}\left(x_{0}\right) \subset D$ satisfying $u\left(x_{0}\right)=\max _{x \in \mathcal{N}\left(x_{0}\right)} u>0$. Then at $x_{0}, D^{2} u \leq 0$. Thus $D^{2} u\left(x_{0}\right)+M\left(x_{0}\right) \leq M\left(x_{0}\right)$ and

$$
\operatorname{det}\left[D^{2} u\left(x_{0}\right)+M\left(x_{0}\right)\right] \leq \operatorname{det}\left[M\left(x_{0}\right)\right]<g_{0} .
$$

This contradicts the equation in (1.2).

Next we prove that $u \neq 0$ in $D$. On the contrary, let some point $x_{0} \in D$ satisfy $u\left(x_{0}\right)=0$. From the above proof, we know that $u\left(x_{0}\right)$ is the maximum of $u$ in $D$. Then at $x_{0}, D^{2} u \leq 0$. We get a contradiction by the similar arguments as the above.

Proof of Theorem 1.1 Let

$$
D^{t}:=D \cap\left\{x_{1} \leq t\right\} \quad(t \leq 0)
$$

Define $u^{t}(x)=u\left(x^{t}\right)$ in $D^{t}$, i.e.,

$$
u^{t}\left(x_{1}, x_{2}, \ldots, x_{n}\right)=u\left(2 t-x_{1}, x_{2}, \ldots, x_{n}\right) \text { in } D^{t}
$$


Then we have

$$
\begin{aligned}
D^{2} u^{t}(x) & =Q D^{2} u\left(2 t-x_{1}, x_{2}, \ldots, x_{n}\right) Q^{T} \\
& =Q D^{2} u\left(x^{t}\right) Q^{T},
\end{aligned}
$$

with $Q=\operatorname{diag}(-1,1, \ldots, 1)$ and $Q^{T}$ being the transpose of $Q$. By the hypothesis $\left(H_{1}\right)$, we know that

$$
M(x)=\left(m_{i j}(x)\right)=Q M\left(x^{t}\right) Q^{T}
$$

Thus

$$
\begin{aligned}
\operatorname{det}\left[D^{2} u^{t}(x)+M(x)\right] & =\operatorname{det}\left[Q D^{2} u\left(x^{t}\right) Q^{T}+Q M\left(x^{t}\right) Q^{T}\right] \\
& =\operatorname{det}\left[\left(D^{2} u+M\right)\left(x^{t}\right)\right] \\
& =g\left(u\left(x^{t}\right)\right) \\
& =g\left(u^{t}(x)\right) .
\end{aligned}
$$

Then

$$
\begin{aligned}
& \log \operatorname{det}\left[D^{2} u(x)+M(x)\right]-\log \operatorname{det}\left[D^{2} u^{t}(x)+M(x)\right] \\
& \quad=\int_{0}^{1} \frac{d}{d \zeta} \log \operatorname{det}\left[\zeta\left(D^{2} u+M\right)+(1-\zeta)\left(D^{2} u^{t}+M\right)\right] d \zeta \\
& \quad=\int_{0}^{1} \bar{h}_{\zeta}^{i j} d \zeta\left(u-u^{t}\right)_{i j} \\
& =F^{i j}\left(u-u^{t}\right)_{i j}
\end{aligned}
$$

where $F^{i j}=\int_{0}^{1} \bar{h}_{\zeta}^{i j} d \zeta, \bar{h}_{\zeta}^{i j}$ is the inverse matrix of $\zeta\left(D^{2} u+M\right)+(1-\zeta)\left(D^{2} u^{t}+M\right)$. So

$$
\begin{aligned}
F^{i j}\left(u-u^{t}\right)_{i j} & =\log g(u)-\log g\left(u^{t}\right) \\
& =\int_{0}^{1} \frac{d}{d \zeta} \log \left(\zeta g(u)+(1-\zeta) g\left(u^{t}\right)\right) d \zeta \\
& =\int_{0}^{1} \frac{1}{\zeta g(u)+(1-\zeta) g\left(u^{t}\right)} d \zeta \frac{g(u)-g\left(u^{t}\right)}{u-u^{t}}\left(u-u^{t}\right) .
\end{aligned}
$$

Set $h^{t}(x)=u(x)-u^{t}(x)$ for $x \in D^{t}$, then $h^{t}$ satisfies

$$
-F^{i j} h_{i j}^{t}+\bar{\theta}(x, t) h^{t}=0, \quad x \in D^{t}
$$

where

$$
\bar{\theta}(x, t)=\int_{0}^{1} \frac{1}{\zeta g(u)+(1-\zeta) g\left(u^{t}\right)} d \zeta \frac{g(u)-g\left(u^{t}\right)}{u-u^{t}} .
$$


From the Lipschitz continuity of $g$, for some constant $\bar{\theta}_{0}>0,|\bar{\theta}(x, t)| \leq \bar{\theta}_{0}$. Since $M$ is independent of $D u$, from $[4,10]$, we know that the equation in (1.2) has the second derivative estimate for the solutions. So $F^{i j}$ is uniformly elliptic.

Obviously, we have $u=u^{t}$ on $\partial D^{t} \cap\left\{x_{1}=t\right\}$. In addition, we know that the reflection of $\partial D \cap \partial D^{t}$ is in the interior of $D$, then, by Lemma 3.1, we get for $t<0, u^{t}<0$ on $\partial D \cap \partial D^{t}$. Thus on $\partial D^{t}, h^{t} \geq 0$. Thanks to Corollary 2.3, we know that $h^{t} \geq 0$ in $D^{t}$ if the width of $D^{t}$ is small enough.

Towards the right, the plane can be moved. Let

$$
t_{0}=\sup \left\{t<0 \mid h^{t} \geq 0 \text { in } D^{t}\right\}
$$

We will prove that

$$
t_{0}=0 .
$$

On the contrary, if $t_{0}<0$, we will demonstrate that the plane can still be moved a little to the right, and this contradicts the definition of $t_{0}$.

Indeed, if $t_{0}<0$, then the image of $\partial D^{t_{0}} \cap \partial D$ through the $\left\{x_{1}=t_{0}\right\}$ reflection is inside $D$. But $u<0$ in $D$ by Lemma 3.1. It follows that on $\partial D^{t_{0}} \cap \partial D, h^{t_{0}}>0$. By the strong maximum principle for the linear elliptic equations,

$$
h^{t_{0}}(x)>0 \text { in } D^{t_{0}} .
$$

Assume that $d_{0}$ is the maximum width of such narrow regions in which we can still utilize the "narrow region principle." Suppose that $\epsilon$ is a small positive constant satisfying $\epsilon<\min \left\{-t_{0}, d_{0} / 2\right\}$. Let the narrow region be

$$
U^{t_{0}+\epsilon}=D^{t_{0}+\epsilon} \cap\left\{x \mid x_{1}>t_{0}-\frac{d_{0}}{2}\right\} .
$$

Now we take into account the function $h^{t_{0}+\epsilon}(x)$ on $U^{t_{0}+\epsilon}$. Similarly to equation (3.2), $h^{t_{0}+\epsilon}(x)$ satisfies

$$
\begin{cases}-\bar{F}^{i j}\left(x, t_{0}+\epsilon\right) h_{i j}^{t_{0}+\epsilon}+\bar{\theta}\left(x, t_{0}+\epsilon\right) h^{t_{0}+\epsilon}=0, & x \in U^{t_{0}+\epsilon}, \\ h^{t_{0}+\epsilon}(x) \geq 0, & x \in \partial U^{t_{0}+\epsilon}\end{cases}
$$

where $\bar{F}^{i j}\left(x, t_{0}+\epsilon\right)=\int_{0}^{1} \bar{g}_{\zeta}^{i j} d \zeta, \bar{g}_{\zeta}^{i j}$ is the inverse matrix of $\zeta\left(D^{2} u+M\right)+(1-\zeta)\left(D^{2} u^{t_{0}+\epsilon}+M\right)$. To see the boundary condition, we first note that it is satisfied on $\partial U^{t_{0}+\epsilon} \cap \partial D$, and on $\partial U^{t_{0}+\epsilon} \cap\left\{x_{1}=t_{0}+\epsilon\right\}$ the same holds, by virtue of the definition of $h^{t_{0}+\epsilon}$. To illustrate that it is also true on $\partial U^{t_{0}+\epsilon} \cap\left\{x_{1}=t_{0}-\frac{d_{0}}{2}\right\}$, we use the continuity argument. If $\left(t_{0}-\frac{d_{0}}{2}, x_{2}, \ldots, x_{n}\right) \in$ $\partial D$, it is clear that

$$
h^{t_{0}+\epsilon}\left(t_{0}-\frac{d_{0}}{2}, x_{2}, \ldots, x_{n}\right) \geq 0 .
$$

If $\left(t_{0}-\frac{d_{0}}{2}, x_{2}, \ldots, x_{n}\right) \in D^{t_{0}}$, by (3.3), we can get that there is a positive constant $\delta_{0}$ such that

$$
h^{t_{0}}\left(t_{0}-\frac{d_{0}}{2}, x_{2}, \ldots, x_{n}\right) \geq \delta_{0}
$$


Since for $\epsilon$ sufficiently small, $h^{t_{0}}$ is continuous in $t_{0}$, then we have

$$
h^{t_{0}+\epsilon}\left(t_{0}-\frac{d_{0}}{2}, x_{2}, \ldots, x_{n}\right) \geq 0 \text {. }
$$

Therefore the boundary condition in (3.4) holds for such a small $\epsilon$. Now applying Corollary 2.3, we get

$$
h^{t_{0}+\epsilon}(x) \geq 0 \quad \text { in } U^{t_{0}+\epsilon}
$$

Consequently, $h^{t_{0}+\epsilon}(x) \geq 0$ in $D^{t_{0}+\epsilon}$ which contradicts the definition of $t_{0}$.

Thus $t_{0}=0$, particularly $h^{0} \geq 0$ in $D^{0}$. This implies for $x_{1}<0$ that

$$
u\left(-x_{1}, x_{2}, \ldots, x_{n}\right) \leq u\left(x_{1}, \ldots, x_{n}\right)
$$

If the plane is moved from right to left, then similarly

$$
u\left(x_{1}, \ldots, x_{n}\right) \leq u\left(-x_{1}, x_{2}, \ldots, x_{n}\right)
$$

It follows that

$$
u\left(x_{1}, \ldots, x_{n}\right)=u\left(-x_{1}, x_{2}, \ldots, x_{n}\right) .
$$

This implies that $u$ is symmetric about the plane $\left\{x: x_{1}=0\right\}$.

For the sake of completing the proof of Theorem 1.1, it is needed to prove that $u$ is decreasing in $x_{1}<0$. In fact, $h^{t}=0$ on the plane $\left\{x_{1}=t, t<0\right\}$, and $h^{t}>0$ on $\partial D \cap \partial D^{t}$. Due to the strong maximum principle, $h^{t}(x)>0$ in $D^{t}$. We then apply the Hopf lemma and conclude that

$$
2 u_{x_{1}}=\frac{\partial h^{t}}{\partial x_{1}}<0 .
$$

Hence Theorem 1.1 is proved.

Remark 3.1 If $g(u)=e^{l(u)}$ in (1.2), where $l(u)$ is Lipschitz continuous and nonnegative in $\left[u_{0}, 0\right], u_{0}=\inf u$, then $\log g(u)=l(u), \log g\left(u^{t}\right)=l\left(u^{t}\right)$ in (3.1), and Theorem 1.1 is still true.

According to Theorem 1.1, if we let the $x_{1}$ axis be any direction, then we obtain

Corollary 3.2 Let $D$ be a ball and $u \in C^{3}(\bar{D})$ be any solution of (1.2). Then $u$ is radially symmetric about the origin and monotone increasing along the radii.

\section{Existence and nonexistence results of radial solutions in a ball}

Proof of Theorem 1.2 (1) For $0<\kappa<1$, from Corollary 3.2, we know that the solution of (1.3) is radially symmetric. Then let $u(x)=u(r), r=|x|$. In addition, $u^{\prime}(r)>0$. Then $u(0) \leq u(x), x \in B_{H}$, and $u^{\prime}(0)=0$. Since $u(H)=0$, one has $u(0)=-C<0$. By a simple calculation,

$$
u_{i}=\frac{x_{i}}{r} u^{\prime}(r)
$$




$$
u_{i j}=\frac{x_{i} x_{j}}{r^{2}} u^{\prime \prime}(r)+\left(\frac{\delta_{i j}}{r}-\frac{x_{i} x_{j}}{r^{3}}\right) u^{\prime}(r) .
$$

The eigenvalues of $D^{2} u+\kappa I$ are $\kappa+u^{\prime \prime}(r), \kappa+\frac{u^{\prime}(r)}{r}, \ldots, \kappa+\frac{u^{\prime}(r)}{r}$, and so

$$
\begin{aligned}
\operatorname{det}\left(D^{2} u+\kappa I\right) & =\left(\kappa+u^{\prime \prime}(r)\right)\left(\kappa+\frac{u^{\prime}(r)}{r}\right)^{n-1} \\
& =\left(\kappa+u^{\prime \prime}(r)\right) \frac{\left(\kappa r+u^{\prime}(r)\right)^{n-1}}{r^{n-1}}
\end{aligned}
$$

Then equation (1.3) becomes

$$
\left(\kappa+u^{\prime \prime}(r)\right) \frac{\left(\kappa r+u^{\prime}(r)\right)^{n-1}}{r^{n-1}}=e^{-u(r)}
$$

Thus

$$
\left(\left(\kappa r+u^{\prime}(r)\right)^{n}\right)^{\prime}=n r^{n-1} e^{-u(r)} .
$$

When $r \in[0, H]$, integrating the above equality on $[0, r]$, we get

$$
\left(\kappa r+u^{\prime}(r)\right)^{n}=\int_{0}^{r} n s^{n-1} e^{-u(s)} d s .
$$

Since $u$ is increasing in $[0, H]$, we get

$$
\begin{aligned}
\left(\kappa r+u^{\prime}(r)\right)^{n} & \geq e^{-u(r)} \int_{0}^{r} n s^{n-1} d s \\
& =r^{n} e^{-u(r)} .
\end{aligned}
$$

It means that

$$
\begin{aligned}
& r e^{-u(r) / n} \leq \kappa r+u^{\prime}(r), \\
& e^{u(r) / n} u^{\prime}(r) \geq r-\kappa r e^{u(r) / n} .
\end{aligned}
$$

Since $0=u(H)>u(r), r \in[0, H]$, then $1=e^{u(H) / n}>e^{u(r) / n}$, so

$$
e^{u(r) / n} u^{\prime}(r) \geq\left(1-\kappa e^{u(H) / n}\right) r=(1-\kappa) r .
$$

That is,

$$
\left(e^{u(r) / n}\right)^{\prime} \geq \frac{r}{n}(1-\kappa) .
$$

By integrating the above inequality on $[0, r]$, we have

$$
e^{u(r) / n}-e^{u(0) / n} \geq \frac{1-\kappa}{2 n} r^{2}
$$


Set $r=H$, then

$$
e^{u(H) / n}-e^{u(0) / n} \geq \frac{H^{2}}{2 n}(1-\kappa) .
$$

Therefore,

$$
H^{2} \leq 2 n\left(1-e^{u(0) / n}\right) /(1-\kappa) .
$$

So if $H$ is large enough, (1.3) has no solution.

(2) If $0<\kappa<e$, define, for $x \in B_{H}$,

$$
\underline{u}(x)=\frac{n\left(|x|^{2}-H^{2}\right)}{H^{2}} .
$$

Then

$$
\operatorname{det}\left(D^{2} \underline{u}+\kappa I\right)=\left(\frac{2 n}{H^{2}}+\kappa\right)^{n} .
$$

Therefore, if $H \leq \sqrt{\frac{2 n}{e-\kappa}}$, we then have

$$
\operatorname{det}\left(D^{2} \underline{u}+\kappa I\right) \geq e^{n} \geq e^{-\underline{u}} .
$$

Thus $\underline{u}(x)$ is one subsolution to (1.3). Due to Remark 4.5 in [21], we know that (1.3) has a unique elliptic solution.

(3) If $\kappa \geq e$ and $0<H<+\infty$ is arbitrary, we still define $\underline{u}(x)$ as above. By (4.3), we know that

$$
\operatorname{det}\left(D^{2} \underline{u}+\kappa I\right) \geq e^{n} \geq e^{-\underline{u}} .
$$

Then for any $H>0, \underline{u}(x)$ is one subsolution to (1.3). As a result, problem (1.3) has a unique elliptic solution.

Remark 4.1 In the proof of Theorem 1.2, (2) and (3), if a subsolution is found, then the existence of solution can be obtained from the global estimates in [35] and an argument similar to the following Lemma 5.3.

\section{Existence and nonexistence results of Hessian equations}

Let $u \in C^{3}(\bar{D})$ be a solution to (1.5). By Theorem 4.2 (see [38]), the equation in (1.5) is uniformly elliptic. Thanks to the Theorem $2.1^{\prime}$ in [23], we have

Theorem 5.1 Suppose that $D$ is symmetric about a hyperplane. Any solution $u \in C^{3}(\bar{D})$ to (1.5) is also symmetric about the hyperplane.

Due to Theorem 5.1, if we let the $x_{1}$ axis be any direction, then we obtain

Corollary 5.2 Let D be a ball. All solutions of (1.5) are radially symmetric with respect to the origin and monotone increasing along the radii. 
Using the above symmetry results, we are able to prove Theorem 1.3. We first apply the upper and lower solution method to build a solution of (1.5) through iteration. We can also refer the reader to [39]. But we will give the proof for the sake of readers' convenience.

Lemma 5.3 Let a uniformly $k$-convex function $\underline{u} \in C^{3}(\bar{D})$ satisfy $S_{k}\left(D^{2} \underline{u}\right) \geq e^{-\underline{u}}$ in $D$ and $\underline{u} \leq 0$ on $\partial D$. Then (1.5) has a solution $u$ in $D$.

Proof Let $u^{0}=\underline{u}$ and

$$
\begin{cases}S_{k}\left(D^{2}\left(u^{k+1}\right)\right)=e^{-u^{k}}, & x \in D, \\ u^{k+1}=0, & x \in \partial D .\end{cases}
$$

By Theorems 3.3 and 3.4 (see [30]), we know that (5.1) has a solution $u^{k+1} \in C^{3, \alpha}(\bar{D})$, and the $C^{1,1}(\bar{D})$ norm of $u^{k}$ can control the $C^{3, \alpha}(\bar{D})$ norm of $u^{k+1}$.

In addition, we have

$$
\begin{cases}S_{k}\left(D^{2}\left(u^{1}\right)\right) \leq S_{k}\left(D^{2}\left(u^{0}\right)\right) & \text { in } D, \\ u^{1} \geq u^{0} & \text { on } \partial D .\end{cases}
$$

Then we have $u^{1} \geq u^{0}$ in $D$ due to the comparison principle. So $e^{-u^{1}} \leq e^{-u^{0}}=S_{k}\left(D^{2}\left(u^{1}\right)\right)$ in $D$. So $u^{k} \leq u^{k+1}$ by induction, and then for any $k, e^{-u^{k+1}} \leq S_{k}\left(D^{2}\left(u^{k+1}\right)\right)$.

Due to Theorem 3.1 (see [30]), the $C^{1,1}(\bar{D})$ norm of $\underline{u}$ can control the $C^{1,1}(\bar{D})$ norm of $u^{k}$. Thus the $C^{1,1}(\bar{D})$ norm of $\underline{u}$ can control the $C^{3, \alpha}(\bar{D})$ norm of $u^{k+1}$ and so the sequence $\left\{u^{k}\right\}$ converges to $u$ in $C^{2}(\bar{D})$. Therefore $u$ is a solution to (1.5) when we take the limit in (5.1).

Proof of Theorem 1.3 According to Corollary 5.2, any solution to (1.5) is radially symmetric. Hence set $u(x)=u(r), r=|x|$. In addition, $u^{\prime}(r)>0$ and $u(0)$ is the minimum of $u$.

Then we have (4.1) and (4.2). A direct calculation gives that

$$
S_{k}\left(D^{2} u\right)=C_{n-1}^{k-1} \frac{r^{1-n}\left(r^{n-k}\left(u^{\prime}\right)^{k}\right)^{\prime}}{k}=e^{-u},
$$

here $C_{n-1}^{k-1}=\frac{(n-1) !}{(k-1) !(n-k) !}$.

From (5.2), we deduce that

$$
\left(r^{n-k}\left(u^{\prime}\right)^{k}\right)^{\prime}=\frac{k}{C_{n-1}^{k-1}} r^{n-1} e^{-u} .
$$

By the integration for $r$ from 0 to $r \in[0, H]$ and using the fact that $u^{\prime}(0)=0$, we have

$$
\left(u^{\prime}\right)^{k} r^{n-k}=\int_{0}^{r} \frac{k}{C_{n-1}^{k-1}} e^{-u(s)} s^{n-1} d s .
$$

Since $u$ is increasing, we know that $e^{-u}$ is then decreasing, and therefore get

$$
\left(u^{\prime}\right)^{k} \geq \frac{k}{n C_{n-1}^{k-1}} r^{k} e^{-u(r)},
$$


that is,

$$
\begin{aligned}
& u^{\prime}(r) \geq\left(\frac{k}{n C_{n-1}^{k-1}}\right)^{\frac{1}{k}} r e^{-u(r) / k}, \\
& \left(\frac{k}{n C_{n-1}^{k-1}}\right)^{\frac{1}{k}} \frac{r}{k} \leq \frac{d}{d r}\left(e^{u(r) / k}\right) .
\end{aligned}
$$

Assume that for some constant $\tilde{C}>0, u(0)=-\tilde{C}$. By integrating with respect to $r$, we derive

$$
e^{u(r) / k} \geq\left(\frac{k}{n C_{n-1}^{k-1}}\right)^{\frac{1}{k}} \frac{r^{2}}{2 k}+e^{-\frac{\tilde{C}}{k}} .
$$

Particularly, we obtain from $u(H)=0$ that

$$
1 \geq\left(\frac{k}{n C_{n-1}^{k-1}}\right)^{\frac{1}{k}} \frac{H^{2}}{2 k}+e^{-\frac{\tilde{C}}{k}} \geq\left(\frac{k}{n C_{n-1}^{k-1}}\right)^{\frac{1}{k}} \frac{H^{2}}{2 k} .
$$

So

$$
H \leq(2 k)^{\frac{1}{2}}\left(\frac{n C_{n-1}^{k-1}}{k}\right)^{\frac{1}{2 k}}
$$

which means that if $H$ is large enough, there does not exist any solution of (1.5).

Let $H>0$ be small enough. To prove that there is a solution of (1.5), we let

$$
u(x)=\frac{n\left(|x|^{2}-H^{2}\right)}{H^{2}} .
$$

Then we have

$$
u_{i j}=\frac{2 n}{H^{2}} \delta_{i j}
$$

So if

$$
H<(2 n)^{\frac{1}{2}}\left(\frac{C_{n}^{k}}{e^{n}}\right)^{\frac{1}{2 k}}
$$

then

$$
S_{k}\left(D^{2} u\right)=C_{n}^{k}\left(\frac{2 n}{H^{2}}\right)^{k} \geq e^{n} \geq e^{-u},
$$

and (5.3) becomes a subsolution of (1.5). Because $u=0$ is always a supsolution of (1.5), then, by Lemma 5.3, there does exist a solution to (1.5) if $H$ is small enough. We thus complete the proof of Theorem 1.3.

Lemma 5.4 Suppose that $D_{1}$ and $D_{2}$ are bounded and uniformly $(k-1)$-convex in $\mathbb{R}^{n}$ satisfying $D_{1} \subset D_{2}$. If the problem (1.5) has a solution $u$ in $D_{2}$, then (1.5) has a solution $v$ in $D_{1}$, or equivalently, if (1.5) has no solution in $D_{1}$, then (1.5) has no solution in $D_{2}$. 
Proof We only need to choose a subsolution to confine $u$ in $D_{1}$ and utilize Lemma 5.3, and then we get the results.

Theorem 5.5 There is a constant $\bar{\mu}_{0}>0$ such that for $\bar{\mu}<\bar{\mu}_{0}$ (1.5) has a solution in $\bar{\mu} D$, while if $\bar{\mu}>\bar{\mu}_{0}$ then (1.5) has no solution in $\bar{\mu} D$.

Proof From Lemma 5.4, we know that if (1.5) has a solution in $\bar{\mu} D$, then for any $\bar{\mu}^{\prime} \in(0, \bar{\mu})$, (1.5) has a solution in $\bar{\mu}^{\prime} D$; and if (1.5) has no solution in $\bar{\mu} D$, then for any $\bar{\mu}^{\prime}>\bar{\mu}$, (1.5) has no solution in $\bar{\mu}^{\prime} D$. Define

$$
\bar{\mu}_{0}=\sup \{\bar{\mu}>0 \mid \text { there is a solution to }(1.5) \text { in } \bar{\mu} D\} .
$$

This completes the proof of Theorem 5.5.

Next we verify Theorem 1.4. Consider the problem

$$
\begin{cases}S_{k}\left(D^{2} \phi\right)=\left|\lambda_{1} \phi\right|^{k}, & x \in D, \\ \phi=0, & x \in \partial D .\end{cases}
$$

Suppose that $\lambda_{1}$ is the first eigenvalue and $\phi$ is the corresponding eigenfunction. Then $\lambda_{1}>0$ and $\phi$ is $k$-convex, $\phi \leq 0$ (see [30]).

Lemma 5.6 If (1.6) has a solution $u$ with $\bar{\alpha}>0$, then $\bar{\alpha}<\lambda_{1}(k !)^{\frac{1}{k}}$.

Proof We argue by contradiction. Assume that (1.6) has a solution $u$ with $\bar{\alpha} \geq \lambda_{1}(k !)^{\frac{1}{k}}$. Let $\phi$ satisfy (5.4). We may suppose that $u(x)<\phi(x)$ for any $x \in D$, otherwise, scaling can be performed if necessary. Let

$$
c^{*}=\sup \{c \mid(u-c \phi)(x) \leq 0, \forall x \in D\} .
$$

Then we can find some point $x_{0} \in D$ satisfying $\left(u-c^{*} \phi\right)\left(x_{0}\right)=0$ and, for $x \in D, u-c^{*} \phi \leq 0$. Set $\tilde{\varphi}=c^{*} \phi$ and consider the linear operator

$$
L=F_{i j}\left(D^{2} \tilde{\varphi}\right) D_{i j}
$$

where $F_{i j}=\partial S_{k}^{\frac{1}{k}} / \partial r_{i j}$. Since $\tilde{\varphi}$ is $k$-convex, using the concavity of $S_{k}^{\frac{1}{k}}$, we conclude that

$$
\begin{aligned}
L(u-\tilde{\varphi}) & \geq S_{k}^{\frac{1}{k}}\left(D^{2} u\right)-S_{k}^{\frac{1}{k}}\left(D^{2} \tilde{\varphi}\right) \\
& =\left(e^{-\bar{\alpha} u}\right)^{\frac{1}{k}}-\left(\left|\lambda_{1} \tilde{\varphi}\right|^{k}\right)^{\frac{1}{k}} .
\end{aligned}
$$

As $-u \geq-\tilde{\varphi} \geq 0$, then we derive that

$$
e^{-\bar{\alpha} u} \geq e^{-\bar{\alpha} \tilde{\varphi}}>\frac{|-\bar{\alpha} \tilde{\varphi}|^{k}}{k !}
$$


So $|\tilde{\varphi}|^{k}<k ! e^{-\bar{\alpha} u}\left(\frac{1}{\bar{\alpha}}\right)^{k}$. Due to $\bar{\alpha} \geq \lambda_{1}(k !)^{\frac{1}{k}}$, we have

$$
\left|\lambda_{1} \tilde{\varphi}\right|^{k}<k ! \lambda_{1}^{k}\left(\frac{1}{\bar{\alpha}}\right)^{k} e^{-\bar{\alpha} u} \leq e^{-\bar{\alpha} u} .
$$

So

$$
0<L(u-\tilde{\varphi}) \quad \text { in } D .
$$

Then we deduce that $u-\tilde{\varphi}$ satisfies

$$
\begin{aligned}
& L(u-\tilde{\varphi})>0, \quad x \in D, \\
& u-\tilde{\varphi}=0, \quad x \in \partial D .
\end{aligned}
$$

Consequently $u-\tilde{\varphi}$ cannot achieve its maximum in $D$. This is a contradiction to the fact that $x_{0}$ is its maximum point in $D$.

From Lemma 5.6, we know that Theorem 1.4 holds.

Lemma 5.7 ([40]) Let $k>n / 2$ and $\alpha=2-n / k$. A $k$-convex function $u$ in $D$ must belong to $C^{\alpha}(D)$.

Theorem 5.8 If $n / 2<k \leq n$, then for the problem (1.6), as $\|u\| \rightarrow \infty$, we have $\bar{\alpha} \rightarrow 0$.

Proof Assume that the sequence $\left\{\left(\bar{\alpha}_{n}, u_{n}\right)\right\}$ satisfies (1.6) and $\left\|u_{n}\right\| \rightarrow \infty, \bar{\alpha}_{n} \rightarrow \bar{\alpha}_{0}>0$, $n \rightarrow \infty$. Let $v_{n}=u_{n} /\left\|u_{n}\right\|$. Then $v_{n}$ satisfies the equation

$$
\begin{aligned}
S_{k}\left(D^{2} v_{n}\right) & =\frac{1}{\left\|u_{n}\right\|^{k}} e^{-\bar{\alpha}_{n} u_{n}} \\
& =\frac{1}{\left\|u_{n}\right\|^{k}} e^{-\bar{\alpha}_{n}\left\|u_{n}\right\| v_{n}} .
\end{aligned}
$$

For any $D_{1} \subset \subset D$, from Lemma 5.7, we know that $\left\|u_{n}\right\| \rightarrow \infty$ uniformly in $D_{1}$. In particular, $\left\|v_{n}\right\| \rightarrow 1$ uniformly in $D_{1}$, which implies that $S_{k}\left(D^{2} v_{n}\right) \rightarrow 0$ for each $x \in D_{1}$.

But, from (5.5) and the fact that $\bar{\alpha}_{n}$ and $v_{n}<0$ are bounded away from zero in $D_{1}$, we derive that in $D_{1}, S_{k}\left(D^{2} v_{n}\right) \rightarrow \infty$. This is a contradiction. Hence $\bar{\alpha} \rightarrow 0$ as $\|u\| \rightarrow \infty$. Thus we complete the proof.

The results of [13] and our results motivate the following conjecture.

Conjecture 1 If $n / 2<k \leq n$, then there is some constant $\bar{\alpha}^{*}>0$ such that, for $\bar{\alpha} \in\left(0, \bar{\alpha}^{*}\right)$, (1.6) has at least two solutions, while for $\bar{\alpha}=\bar{\alpha}^{*},(1.6)$ has a unique solution.

Acknowledgements

Not applicable.

Funding

The research was supported by National Natural Science Foundation of China (No. 11201343) and Shandong Provincial Natural Science Foundation (ZR2018LA006). 
Abbreviations

Not applicable.

\section{Availability of data and materials}

Not applicable.

Ethics approval and consent to participate

Not applicable.

\section{Competing interests}

The authors declare that they have no competing interests.

\section{Consent for publication}

Not applicable.

\section{Authors' contributions}

The first author proposed the idea of this paper and wrote the whole paper. The second author performed all the steps of the proofs. The third author prepared the manuscript initially. All authors read and approved the final manuscript.

\section{Authors' information}

Not applicable.

\section{Author details}

${ }^{1}$ School of Mathematics and Information Science, Weifang University, Weifang, China. ${ }^{2}$ School of Mathematics and Statistics, North China University of Water Resources and Electric Power, Zhengzhou, China. ${ }^{3}$ College of Mathematics and System Science, Shandong University of Science and Technology, Qingdao, China.

\section{Publisher's Note}

Springer Nature remains neutral with regard to jurisdictional claims in published maps and institutional affiliations.

Received: 18 March 2021 Accepted: 18 August 2021 Published online: 08 September 2021

\section{References}

1. Guan, P.F., Wang, X.J.: On a Monge-Ampère equation arising in geometric optics. J. Differ. Geom. 48, 205-222 (1998)

2. Villani, C.: Topics in Optimal Transportation. Graduate Studies in Mathematics, vol. 58. Am. Math. Soc., Providence (2003)

3. Trudinger, N.S.: Recent developments in elliptic partial differential equations of Monge-Ampère type. In: International Congress of Mathematicians, vol.III, pp. 291-301. Eur. Math. Soc., Zürich (2006)

4. Han, Q., Hong, J.X.: Isometric Embedding of Riemannian Manifolds in Euclidean Spaces. Mathematical Surveys and Monographs, vol. 130. Am. Math. Soc., Providence (2006)

5. Gutiérrez, C.E., Huang, Q.B.: The refractor problem in reshaping light beams. Arch. Ration. Mech. Anal. 193, 423-443 (2009)

6. Gutiérrez, C.E., Huang, Q.B.: The near field refractor. Ann. Inst. Henri Poincaré, Anal. Non Linéaire 31, 655-684 (2014)

7. Karakhanyan, A., Wang, X.J.: On the reflector shape design. J. Differ. Geom. 84, 561-610 (2010)

8. Wang, X.J.: On the design of a reflector antenna. Inverse Probl. 12, 351-375 (1996)

9. Caffarelli, L.A.: Non linear elliptic theory and the Monge-Ampère equation. In: Proceedings of the International Congress of Mathematicians, vol. I (Beijing, 2002), pp. 179-187. Higher Ed. Press, Beijing (2002)

10. Trudinger, N.S., Wang, X.J.: The Monge-Ampère equation and its geometric applications. In: Handbook of Geometric Analysis, No. 1. Adv. Lect. Math. (ALM), vol. 7, pp. 467-524. Int. Press, Somerville (2008)

11. Caffarelli, L.A., Nirenberg, L., Spruck, J.: The Dirichlet problem for nonlinear second-order elliptic equations, I. Monge-Ampère equation. Commun. Pure Appl. Math. 37, 369-402 (1984)

12. Krylov, N.V.: Boundedly inhomogeneous elliptic and parabolic equations in a domain. IzV. Akad. Nauk SSSR, Ser. Mat. 47, 75-108 (1983) (Russian)

13. Zhang, Z.T., Wang, K.L.: Existence and non-existence of solutions for a class of Monge-Ampère equations. J. Differ. Equ. 246, 2849-2875 (2009)

14. Zhang, X.M., Feng, M.Q.: Boundary blow-up solutions to the Monge-Ampère equation: sharp conditions and asymptotic behavior. Adv. Nonlinear Anal. 9(1), 729-744 (2020)

15. Mohammed, A., Rădulescu, V.D., Vitolo, A.: Blow-up solutions for fully nonlinear equations: existence, asymptotic estimates and uniqueness. Adv. Nonlinear Anal. 9(1), 39-64 (2020)

16. Feng, M.Q.: Convex solutions of Monge-Ampère equations and systems: existence, uniqueness and asymptotic behavior. Adv. Nonlinear Anal. 10(1), 371-399 (2021)

17. Ma, X.N., Trudinger, N.S., Wang, X.J.: Regularity of potential functions of the optimal transportation problem. Arch. Ration. Mech. Anal. 177, 151-183 (2005)

18. Trudinger, N.S., Wang, X.J.: On the second boundary value problem for Monge-Ampère type equations and optima transportation. Ann. Sc. Norm. Super. Pisa, Cl. Sci. 8, 143-174 (2009)

19. Liu, J.K., Trudinger, N.S., Wang, X.J.: Interior $C^{2, \alpha}$ regularity for potential functions in optimal transportation. Commun. Partial Differ. Equ. 35, 165-184 (2010)

20. Huang, Y., Jiang, F.D., Liu, J.K.: Boundary $C^{2, \alpha}$ estimates for Monge-Ampère type equations. Adv. Math. 281, 706-733 (2015)

21. Jiang, F.D., Trudinger, N.S., Yang, X.P.: On the Dirichlet problem for Monge-Ampère type equations. Calc. Var. Partial Differ. Equ. 49, 1223-1236 (2014) 
22. Dai, L.M., Li, H.Y.: Entire subsolutions of Monge-Ampère type equations. Commun. Pure Appl. Anal. 19, 19-30 (2020)

23. Gidas, B., Ni, W.M., Nirenberg, L.: Symmetry and related properties via the maximum principle. Commun. Math. Phys. 68, 209-243 (1979)

24. Alexandrov, A.D.: A characteristic property of spheres. Ann. Mat. Pura Appl. 58, 303-315 (1962)

25. Serrin, J.: A symmetry problem in potential theory. Arch. Ration. Mech. Anal. 43, 304-318 (1971)

26. Li, C.: Monotonicity and symmetry of solutions of fully nonlinear elliptic equations on unbounded domains Commun. Partial Differ. Equ. 16, 585-615 (1991)

27. Dai, L.M.: Symmetry of viscosity solutions for fully nonlinear parabolic equations. Nonlinear Anal., Real World Appl. 29 68-79 (2016)

28. Caffarelli, L.A., Nirenberg, L., Spruck, J.: The Dirichlet problem for nonlinear second-order elliptic equations. III. Functions of the eigenvalues of the Hessian. Acta Math. 155, 261-301 (1985)

29. Dai, L.M., Bao, J.G.: On uniqueness and existence of viscosity solutions to Hessian equations in exterior domains. Front. Math. China 6, 221-230 (2011)

30. Wang, X.J.: The k-Hessian equation. In: Geometric Analysis and PDEs. Lecture Notes in Math., vol. 1977, pp. 177-252. Springer, Dordrecht (2009)

31. Lions, P.L.: On the existence of positive solutions of semilinear elliptic equations. SIAM Rev. 24, $441-467$ (1982)

32. Wang, X.J.: Existence of multiple solutions to the equations of Monge-Ampère type. J. Differ. Equ. 100, 95-118 (1992)

33. Jacobsen, J.: Global bifurcation problems associated with K-Hessian operators. Topol. Methods Nonlinear Anal. 14 81-130 (1999)

34. Dai, L.M.: Multiple solutions to Hessian equations. Manuscr. Math. 134, 423-433 (2011)

35. Li, Y.Y.: Some existence results for fully nonlinear elliptic equations of Monge-Ampère type. Commun. Pure Appl. Math. 43, 233-271 (1990)

36. Wang, B., Bao, J.G.: Mirror symmetry for a Hessian over-determined problem and its generalization. Commun. Pure Appl. Anal. 13, 2305-2316 (2014)

37. Wang, B., Bao, J.G.: Over-determined problems for k-Hessian equations in ring-shaped domains. Nonlinear Anal. 127, 143-156 (2015)

38. Chou, K.S., Wang, X.J.: A variational theory of the Hessian equation. Commun. Pure Appl. Math. 54, 1029-1064 (2001)

39. Wang, X.J.: A class of fully nonlinear elliptic equations and related functionals. Indiana Univ. Math. J. 43, 25-54 (1994)

40. Trudinger, N.S., Wang, X.J.: Hessian measures. II. Ann. Math. 150, 579-604 (1999)

\section{Submit your manuscript to a SpringerOpen ${ }^{\circ}$ journal and benefit from:}

- Convenient online submission

Rigorous peer review

Open access: articles freely available online

- High visibility within the field

- Retaining the copyright to your article

Submit your next manuscript at springeropen.com 